\title{
Cláudio Tinoco Mesquita and Maria Fernanda Rezende (eds.). Nuclear Cardiology: Basic and Advanced Concepts in Clinical Practice
}

\author{
Springer Nature Switzerland AG 2021, 781 pp., ISBN \\ 978-3-030-62194-0 ISBN 978-3-030-62195-7 \\ (eBook); hardcover $155.99 €$, e-book $119.99 €$
}

\author{
Wael AlJaroudi, MD, FASNC \\ a Division of Cardiovascular Medicine, Augusta University-Medical College of Georgia, Augusta, \\ GA
}

Received Apr 29, 2021; accepted Apr 29, 2021

doi: $10.1007 / \mathrm{s} 12350-021-02664-0$

\section{INTRODUCTION}

The field of nuclear cardiology has witnessed exponential growth in the last decade, from advancement in cutting edge technology, tracers, hybrid imaging, new clinical and research applications, key clinical trials, and artificial intelligence. Similarly, the application of nuclear cardiology has expanded with more diagnostic, preventive, prognostic, and guidance of clinical decision making. Not surprisingly, there have been countless studies, publications, editorials, ongoing research dispersed throughout the literature, journals, and meeting discussions, that spread through the field of nuclear imaging and transcend it to other non-invasive modalities. Indeed, nuclear cardiology has become a unique specialty, as it is now customary to integrate cardiac computed tomography (CCT) and magnetic resonance (CMR) as complementary modalities or hybrid imaging in daily clinical applications, research, publications, and in society meetings.

Keeping up with all the advancement in the field can be challenging, even to those who are most experienced. Nuclear Cardiology: Basic and Advanced Concepts in Clinical Practice (Cláudio Tinoco Mesquita and Maria Fernanda Rezende (eds.). Springer Nature

Reprint requests: Wael AlJaroudi, MD, FASNC, Division of Cardiovascular Medicine, Augusta University-Medical College of Georgia, BB-6520B1120 15th St, Augusta, GA 30912; waljaroudi@augusta.edu

J Nucl Cardiol 2021;28:1775-80.

$1071-3581 / \$ 34.00$

Copyright () 2021 American Society of Nuclear Cardiology.
Switzerland AG 2021) could not have come at a better time. The book is a comprehensive, distilled, and up to date summary of the nuclear cardiology literature; it reviews the pathophysiology of heart disease and all aspects where nuclear cardiology plays a pivotal role in 33 dedicated chapters. While some of the chapters are classic topics with updated data, there are many fascinating chapters rarely found in other nuclear cardiology textbooks. In fact, the dedicated topics such as nuclear imaging in the era of COVID-19, artificial intelligence, complementary multimodality imaging with CCT angiogram, calcium score, CMR, and hybrid imaging, are some of the many highlights of book.

The current textbook is not an updated version of a prior edition, but rather a new book with a fresh look at the growing and changing field of nuclear cardiology, enriched with significantly new material, and with a look toward the future. It is written for the benefit of the trainee, but rich and detailed enough for the established providers, including internist, cardiologists, and imaging specialists.

\section{CHAPTER 1: CARDIAC PET PROCEDURE: PERFUSION, CORONARY FLOW, VIABILITY, INFLAMMATION, AND PET/MR}

The chapter is a comprehensive review about cardiac positron emission tomography (PET), from technique, tracers, perfusion, flow, viability, infiltrative, inflammatory and infectious diseases, and with an overview on hybrid PET/MR. The "key points paragraphs" that summarize the takeaway messages, the 
comparative tables, as well as the well-thought illustrative cases are some of the many highlights of the chapters.

\section{CHAPTER 2: SPECT PROCEDURES}

The chapter is a distilled summary of the different types of stress testing commonly performed in nuclear cardiology (exercise, pharmacological and hybrid); their indications and contraindications; and stress testing in special cohorts. It also provides the reader with comparative tables of the different pharmacological agents, the commonly used radioactive SPECT tracers, as well as detailed workflow diagrams.

\section{CHAPTER 3: EVALUATION OF MYOCARDIAL BLOOD FLOW AND MYOCARDIAL FLOW RESERVE BY RADIONUCLIDE IMAGING}

The chapter focuses on the role of PET in evaluating myocardial blood flow and flow reserve, starting with the technique, tracers, and workflow dynamics. The well-designed illustrative cases ensure that the concepts are well grasped and highlight the clinical value of PET.

\section{CHAPTER 4: RADIATION PROTECTION AND EXPOSURE IN NUCLEAR CARDIOLOGY}

The chapter is a classic review of radiation exposure in nuclear cardiology, yet remains of outmost importance in our daily work. It emphasizes the importance of recognizing radiation impact on the patient, the means to minimize and reduce it, and the necessary precautions to ensure the staff well-being.

\section{CHAPTER 5: NON-NUCLEAR CARDIAC IMAGING MODALITIES: CT AND MRI}

The chapter reviews the role of non-nuclear cardiac imaging modalities, particularly cardiac magnetic resonance (CMR) and computed tomography (CCT) in assessing cardiomyopathy, viability, and coronary artery disease. This is a unique and up to date chapter, rarely encountered in traditional nuclear cardiology textbooks, that highlights the complementary role of CMR and CCT to nuclear imaging. It is a great chapter for fellows, internist, general cardiologist, and imaging specialists. It sets the tone for the future of hybrid imaging with PET/ $\mathrm{MR}$ and PET/CT

\section{CHAPTER 6: CORONARY PHYSIOLOGY: FROM BASIC CONCEPTS TO FFR AND IFR}

This is a comprehensive chapter that explains that coronary physiology of flow reserve, starting from basic concepts to mechanism of pathology, clinical application, and prognostic value. Also, it introduces the concept of instantaneous wave-free ratio (iFR) which is a new concept in quantifying flow reserve and stenosis. Finally, the highlight of the chapter are the many illustrative cases that put these concepts into practical application, not to mention how to deal with result discrepancy.

\section{CHAPTER 7: CORONARY ARTERY CALCIUM AND HYBRID IMAGING IN ISCHEMIC HEART DISEASE}

The chapter is a unique addition to the nuclear cardiology books as it addresses in great depth coronary artery calcium (CAC) score in conjunction with myocardial perfusion imaging (MPI), whether calcification detected on attenuation-corrected images, dedicated CT CAC score, or full CCT angiogram imaging, ultimately ending with fusion and hybrid imaging. The added value of coronary artery calcification in terms of diagnostic accuracy, clinical decision management, and prognostication is truly fascinating.

\section{CHAPTER 8: CORONARY BLOOD FLOW RESERVE AND MYOCARDIAL ISCHEMIA}

The chapter summarizes coronary and myocardial blood flow reserve, from physiology to pathophysiology, tracers, PET imaging techniques, and workflow. It complements chapters 3 and 6 and expands into the growing role of myocardial blood flow with SPECT imaging.

\section{CHAPTER 9: NUCLEAR CARDIOLOGY AND CORONAVIRUS DISEASE 2019 (COVID- 19) PANDEMIC}

This is a unique and one of the first comprehensive chapters that tackles the cardiovascular complications of COVID-19, the impact of the pandemic on daily practice for general cardiologists and nuclear imaging specialists, the shift toward telehealth medicine, creative triaging and stratification, and recommendations for best practice. The authors and editors should be congratulated for their ability to compile much data and write up a dedicated chapter in the midst of the pandemic. 


\section{CHAPTER 10: NUCLEAR IMAGING IN STABLE ISCHEMIC CORONARY DISEASE}

The chapter summarizes the role of SPECT and PET imaging in patients with stable coronary artery disease, their clinical and prognostic value, as well their role in special cohorts including diabetes, women, elderly, multivessel disease, and athletes (newly recognized and discussed entity with more data).

\section{CHAPTER 11: NUCLEAR IMAGING IN ACUTE CORONARY SYNDROMES}

The chapter illustrates the role of SPECT and PET imaging in acute coronary syndrome and mimickers (Takotsubo-also discussed in more details in chapter 19). It covers workflow, imaging techniques, assessment of salvage index, clinical and prognostic utility, as well as guidelines approach. The embedded teaching cases and images are truly fascinating and are one of the highlights of the chapter.

\section{CHAPTER 12: NUCLEAR TECHNIQUES BEFORE AND AFTER CORONARY REVASCULARIZATION}

The chapter reviews the value of myocardial perfusion imaging pre and post coronary revascularization, discusses appropriateness criteria and related guidelines, and blends the concepts though illustrative cases encountered in daily clinical practice.

\section{CHAPTER 13: PHYSIOLOGIC AND/ OR ANATOMIC ASSESSMENT OF CAD: PATIENT- CENTERED APPROACH}

The chapter is mind stimulating as it journeys through the ongoing debate between physiologic testing with MPI versus anatomic assessment with CCT angiography. It portrays the two spectrums, enriches the reading with illustrative cases, and prepares the reader to concept of hybrid and fusion imaging.

\section{CHAPTER 14: EVIDENCE-BASED AND NUCLEAR CARDIOLOGY}

The chapter is a selection of the major key trials in nuclear imaging spanning acute and chronic coronary syndromes, heart failure, viability, neuronal imaging, among others. It is a distillation of the clinical data and evidence-based medicine that shaped the current imaging guidelines, well summarized and available for review in a unique single chapter for easy access.

\section{CHAPTER 15: PATHOPHYSIOLOGY, DIAGNOSIS, AND MANAGEMENT OF HEART FAILURE}

The chapter describes the pathophysiology of heart failure, remodeling, types of cardiomyopathy, and clinical approach to diagnosis and management. It is a nice review for trainees and cardiologists, as it sets the ground to going in depth into the nuclear imaging of cardiomyopathies.

\section{CHAPTER 16: PET AND SPECT EVALUATION OF VIABLE DYSFUNCTIONAL MYOCARDIUM}

The chapter discusses the pathophysiology of hibernating myocardium, the concepts that led to viability assessment, and the different imaging techniques and tracers with SPECT and PET. It also covers in depth the clinical and prognostic value of nuclear evaluation of viable myocardium, not to mention key trials that are enrolling and will answer remaining burning questions.

\section{CHAPTER 17: BONE TRACERS FOR THE DIAGNOSIS OF CARDIAC AMYLOIDOSIS}

In the last few years, there has been exponential growth of data supporting the role of nuclear imaging in cardiac amyloidosis using bone tracers. The chapter is a comprehensive summary of the pathophysiology of cardiac amyloidosis and its different subtypes, indication for testing, imaging protocols and techniques with SPECT imaging, as well as the potential role for PET imaging and other tracers.

\section{CHAPTER 18: ADRENERGIC NERVOUS SYSTEM IMAGING IN HF MANAGEMENT}

The chapter reviews neuronal SPECT imaging with MIBG tracer, starting from the pathophysiology of myocardial innervation in heart failure, MIBG kinetics and washout rate, and most recent clinical data for risk stratification and prognostication. It also touches up on potential PET tracers for neuronal imaging in heart failure and emphasizes area of potential research.

\section{CHAPTER 19: TAKOTSUBO CARDIOMYOPATHY AND NUCLEAR IMAGING}

The chapter is a dedicated review of Takotsubo cardiomyopathy and illustrates the complementary role of SPECT Tc-99m and MIBG imaging for diagnosis and risk stratification. 


\section{CHAPTER 20: PET AND SPECT \\ IN INFLAMMATORY DISEASES: SARCOIDOSIS, MYOCARDITIS, AND VASCULITIS}

The chapter is a condensed summary of the role of SPECT, PET, and hybrid PET/MR in main inflammatory diseases, namely sarcoidosis, myocarditis, and vasculitis. It covers the pathology, patient preparation, test interpretation and pitfalls, emerging new tracers, and future research.

\section{CHAPTER 21: CARDIOVASCULAR RISK STRATIFICATION PRIOR TO NON-CARDIAC SURGERY}

The chapter is classic for trainee, internists, and cardiologists. It never gets old to read, particularly when it is so well written and updated. It offers a nice summary on the importance of risk stratification and the selected role of nuclear imaging in that regard.

\section{CHAPTER 22: EXERCISE AND PHARMACOLOGICAL STRESS TESTING}

The chapter is another classic chapter for trainee and cardiologist: condensed yet comprehensive summary about exercise and pharmacological stress testing, from physiology, to the test itself, optimization, and appropriate use criteria.

\section{CHAPTER 23: EVALUATION OF VENTRICULAR FUNCTION BY NUCLEAR IMAGING}

The chapter describes role of nuclear imaging in assessing and quantifying ventricle volume, systolic function, as well as diastolic filling patterns and properties using classic blood pool technique and gated SPECT imaging, not to forget the clinical implication in terms of prognostic value. The mechanical dyssynchrony is discussed in more details in chapter 25.

\section{CHAPTER 24: 123I-MIBG IN THE RISK STRATIFICATION OF SUDDEN CARDIAC DEATH IN CHRONIC HEART FAILURE}

The chapter is complementary to chapter 18 , offers a comprehensive overview on the role of 123I-mIBG in heart failure, covering the pathophysiology, technical aspect of test performance, and how best to interpret the images. It also illustrates the practical aspect of mIBG imaging in risk stratifying for sudden cardiac death and guiding appropriate therapy and clinical decision making.

\section{CHAPTER 25: NUCLEAR IMAGE-GUIDED METHODS FOR CARDIAC RESYNCHRONIZATION THERAPY}

The chapter is a dedicated summary of the role of nuclear imaging in guiding decision for cardiac resynchronization therapy, which is a topic of great interest to most cardiologists and particularly electrophysiologists. It emphasizes the importance of extracting key data from the nuclear study such as ejection fraction, viability, mechanical dyssynchrony, and latest onset of mechanical contraction by phase analysis to optimize patient selection for resynchronization. It also discusses the potential role of artificial intelligence and complex neural network algorithm to further refine the predictive model. The numerous illustrative cases are truly fascinating and reinforce the concepts.

\section{CHAPTER 26: NUCLEAR IMAGING IN THE MANAGEMENT OF INFECTIVE ENDOCARDITIS}

The chapter is a condensed summary of the role of nuclear in detecting infective endocarditis using the traditional leukocyte tagging scintigraphy, as well as PET imaging with new tracers.

\section{CHAPTER 27: PET AND SPECT IN THE EVALUATION OF CARDIAC IMPLANTABLE ELECTRONIC DEVICES}

The chapter is a continuation of the prior one. It discusses the role of nuclear imaging and the complementary role of other non-nuclear imaging modalities in diagnosing and managing patients with cardiac implantable electronic device infection. The readers will enjoy the numerous cases and multimodality images.

\section{CHAPTER 28: THE CLINICAL ASPECTS OF HEART DAMAGE BY CHEMOTHERAPY AND RADIOTHERAPY}

The chapter discusses chemotherapy and radiationinduced cardiomyopathy from a clinical perspective in a nice well-integrated summary that serves as an introduction to the following chapter. 


\section{CHAPTER 29: NUCLEAR MEDICINE TOOLS FOR CARDIAC DAMAGE DIAGNOSIS IN ONCOLOGY}

Cardio-oncology is an emerging specialty on its own with significant contribution from nuclear cardiology. The chapter discusses and compares the various nuclear imaging modalities and their complementary role, including MUGA, SPECT, MIBG, PET imaging for perfusion, metabolism and inflammation, apoptosis, and receptor imaging-targeted imaging. The chapter is a great resource for heart failure and cardio-oncology specialists.

\section{CHAPTER 30: ACUTE CORONARY SYNDROME EVALUATION WITH NUCLEAR MEDICINE IN THE EMERGENCY SETTING}

The chapter summarizes the role of imaging for patients presenting to the emergency room with chest pain. It discusses both nuclear and non-nuclear imaging modalities and focuses on the advancement and progress made with SPECT and PET imaging, as well a nice practical workflow algorithm to guide clinicians.

\section{CHAPTER 31: DIAGNOSIS OF PULMONARY EMBOLISM}

The chapter is a new concept, often ignored in many textbooks. Patients with pulmonary embolism often present with chest pain and positive troponin. While nuclear cardiologists rarely read ventilation-perfusion (V/Q) scans, the chapter is a nice informative summary that is easy to read on role of V/Q in comparison to CT angiogram, indications, advantages and limitations, as well as an integrated work algorithm and nice illustrative cases.

\section{CHAPTER 32: DEEP LEARNING AND ARTIFICIAL INTELLIGENCE IN NUCLEAR CARDIOLOGY}

The field of radiomics is growing exponentially. This chapter brings to light a new field unknown to many, and rarely discussed in other nuclear books. It defines the role of artificial intelligence in nuclear cardiology and illustrates how it is being integrated in the field, starting with providing robust data analysis through artificial neural network, improving diagnostic accuracy, and predictive modeling leading to better clinical risk stratification and management of patients.

\section{CHAPTER 33: PROGRAMS OF THE INTERNATIONAL ATOMIC ENERGY AGENCY FOR NUCLEAR CARDIOLOGY AND QUALITY MANAGEMENT}

The chapter gives credit to the efforts set by the International Atomic Energy Agency and other societies in the service of the field, to promote nuclear cardiology worldwide, provide supportive role, while highlighting cost-effective strategies and decision makings behind many guidelines and quality management. Furthermore, the chapter discusses the challenges the agency faces with new technologies, having to weigh the benefits of technical, diagnostic, therapeutic, prognostic, and social efficacy; hence, outlining the importance of hierarchy of thinking on a higher level, and put things into perspective for clinicians.

\section{PERSPECTIVE}

The book by Cláudio Tinoco Mesquita and Maria Fernanda Rezende (eds.) is a comprehensive summary of the nuclear cardiology literature, condensed in 33 chapters with more than 2000 references. The amount of work, time, and effort invested in the book are simply enormous. To put things into perspective, the editors have enlisted the combined efforts of biologists, physicists, scientists, engineers, medical doctors, PhDs, and data analysts; 75 authors (49 from Brazil) from 12 countries and 4 continents worked tirelessly with the editors to produce a unique book that summarizes the entire nuclear literature, spanning the past, present, and looking toward the future.

While there are some redundancies in the material covered in several chapters, this is not unexpected, especially when you have 75 authors as main contributors. Moreover, each chapter is written in such a way that it can be read independently and with the needed background and necessary details; still, the book flows well as a story. In addition, the readers would enjoy the different styles of the authors and their perspective, giving a broader and multinational view to the topics.

One of the distinguishing features of the book is that it is inclusive of other complementary non-nuclear modalities, gives them credit, and emphasizes the role of hybrid imaging that has become a reality. Furthermore, the book is inclusive of nuclear practice around the world. Although the majority of the authors are from Brazil, the remaining authors are spread across 4 continents, making the data and experience truly comprehensive and enriching.

The book includes classic well written and updated chapters on physiology, radiation safety, preoperative assessment, and many others. Yet, the editors have 
introduced unique and well-thought chapters such as nuclear imaging in the era of COVID-19, the role of artificial intelligence, coronary calcification and role of CT angiography, CMR and hybrid imaging, resynchronization therapy, and mechanical dyssynchrony. There is also a dedicated chapter summarizing the key clinical imaging trials. The selection of topics and design of chapters was really outstanding as it increased the pool of readers to include trainee, internists, general cardiologist, and imaging specialists (cardiologists and radiologists). Even specialized non-imaging cardiologists such as interventionalist, heart failure, and electrophysiologist would enjoy reading several of the chapters that are relevant to their practice. Moreover, the book is available in hard and soft copies, written in English and in Spanish, therefore, able to reach millions of readers worldwide.

In the preface, the editor Dr Mesquita revealed: "During my cardiology training, a special friend, Marcílio Henrique de Souza, gave me a seminal book edited by Dr. Mario Verani and Dr. Ami Iskandrian, Nuclear Cardiac Imaging, Principles and Applications (1996). I have read that book many times and it convinced me to specialize in nuclear cardiology. I sincerely hope that this book we are presenting here, Nuclear Cardiology, Basic and Advanced Concepts in
Clinical Practice (2020), could be an inspiration for many others to follow this path." I can safely say that this book will be an inspiration to this generation and many others. It is also a nice coincidence that the review of this book is being published in the Journal of Nuclear Cardiology, with Dr Ami Iskandrian as the chief editor. Inspiring people to follow a certain path, witnessing their success and their transformation to become leaders in the field, sharing their knowledge and inspiring in return other generations, is truly a heartwarming feeling.

The editors should be congratulated on this enormous task and their ability to gather 75 authorities from across the world to put in all-inclusive, condensed and distilled summary of nuclear cardiology, and with a fair share of complementary imaging modalities. The key messages and summary points, illustrative clinical cases, high-quality images, and workflow diagrams are frankly mesmerizing and one of the many features of the book.

I feel honored and humbled to have been given the opportunity to read and enjoy this book, a review of the work and effort of world authorities in their field.

\section{Disclosure}

Wael AlJaroudi has no disclosures or conflicts of interest. 